\title{
MicroRNA-124-3p inhibits cell growth and metastasis in cervical cancer by targeting IGF2BP1
}

\author{
PING WANG ${ }^{1}$, LI ZHANG ${ }^{1}$, JUNHUI ZHANG ${ }^{1}$ and GANGSHU XU ${ }^{2}$ \\ Departments of ${ }^{1}$ Gynaecology and Obstetrics, and ${ }^{2}$ Neurosurgery, \\ Dezhou People's Hospital, Dezhou, Shandong 253014, P.R. China
}

Received October 24, 2016; Accepted June 15, 2017

DOI: $10.3892 /$ etm.2017.5528

\begin{abstract}
MicroRNAs (miRs) serve a role in promoting and suppressing tumors in various types of malignant cancer, such as cervical cancer. However, the regulatory mechanism of miR-124-3p in cervical cancer remains unclear. In the present study, miR-124-3p was significantly downregulated in cervical cancer tissues and cell lines compared with matching adjacent non-tumor tissues and the normal cervical epithelial cell line End1/E6E7, respectively. Decreased expression of miR-124-3p was associated with advanced cervical cancer and the results of an in vitro study demonstrated that the ectopic expression of miR-124-3p significantly decreased the proliferation, migration and invasion of cervical cancer Caski cells. Furthermore, insulin-like growth factor 2 mRNA binding protein 1 (IGF2BP1) was identified as a novel target of miR-124-3p. Overexpression of miR-124-3p decreased the expression of IGF2BP1, whereas miR-124-3p knockdown promoted IGF2BP1 expression at the post-transcriptional level in Caski cells. Additionally, overexpression of IGF2BP1 attenuated the suppressive effects of miR-124-3p on the proliferation, migration and invasion of Caski cells. IGF2BP1 was upregulated in cervical cancer tissues and cell lines compared with matching adjacent non-tumor tissues and the End1/E6E7 cell line, respectively. Therefore, the present study suggests that miR-124-3p suppresses the growth and metastasis of cervical cancer by directly targeting IGF2BP. Thus, miR-124-3p may be developed as a novel method of treating cervical cancer.
\end{abstract}

\section{Introduction}

Cervical cancer is one of the most common types of cancer diagnosed in women, particularly in developing countries such as China (1). In addition, it is the fourth most frequent cause of

Correspondence to: Professor Li Zhang, Department of Gynaecology and Obstetrics, Dezhou People's Hospital, 1751 Xinhu Street, Dezhou, Shandong 253014, P.R. China

E-mail: dezhouzhangli@aliyun.com

Key words: cervical cancer, microRNA, proliferation, migration, invasion, insulin-like growth factor 2 mRNA binding protein 1 cancer-associated mortality in females (1). Despite improvements in the diagnosis and treatment of cervical cancer, the prognosis of patients with adyanced cervical cancer remains poor (2). Therefore, identifying the molecular mechanism underlying the growth and metastasis of cervical cancer is critical to facilitate the development of novel therapeutic strategies.

MicroRNAs (miRs) are a class of non-coding RNAs that are 18-25 nucleotides long. They function as regulators of gene expression by directly binding to 3'-untranslated regions (UTRs) of target mRNAs, leading to mRNA degradation or the inhibition of protein translation. Through inhibiting the expression of their target mRNAs, miRs have been implicated in various biological processes, including cell survival, proliferation, differentiation, migration and invasion. It has been demonstrated that miRs serve a role in the promotion and suppression of tumors during the development and progression of malignant cervical cancer (3). For example, miR-27b is upregulated in cervical cancer and promotes cell growth, invasion and the epithelial-mesenchymal transition (EMT) by directly targeting cadherin II (CDH11) (4). Additionally, miR-143, which promotes apoptosis and inhibits tumor formation by targeting $\mathrm{Bcl}-2 \mathrm{k}$, is downregulated in cervical cancer (3).

miR-124 is involved in the regulation of several different types of cancer (5). Han et al (6) identified that miR-124 was downregulated in osteosarcoma and that this decrease in the expression of miR-124 was associated with an advanced clinical stage, distant metastasis, poor response to neoadjuvant chemotherapy and a shorter survival time of patients with osteosarcoma. In addition, An et al (5) identified that miR-124 inhibited the migration and invasion of glioma cells by inhibiting the expression of Rho-associated protein kinase 1 (ROCK1). Furthermore, it has been demonstrated that miR-124-3p serves an inhibitory role in cervical cancer. Wilting et al (7) reported that miR-124 was significantly downregulated in cervical cancer due to high methylation, and downregulation of miR-124 was associated with high-grade lesions. In addition, miR-124 serves a suppressive role in cervical cancer by targeting astrocyte elevated gene-1 protein, ribosome interacting Gtpase (RBG2) and angiomotin-like protein 1 (AmotL1) (8-10). However, it remains unclear whether there are any other target genes of miR-124-3p in cervical cancer. 
Therefore, the present study aimed to determine the exact role and underlying molecular mechanism of miR-124-3p in the growth and metastasis of cervical cancer.

\section{Materials and methods}

Tissue collection. A total of 85 cervical cancer tissues and adjacent non-tumor tissues were collected from Dezhou People's Hospital between March 2012 and September 2014. A total of 85 female patients aged 43-71 years (mean, 58.4 years) were enrolled in the present study. No patients received radiotherapy or chemotherapy prior to surgical resection. The clinicopathological characteristics of the patients are summarized in Table I. All tissues were immediately snap-frozen in liquid nitrogen following surgical removal. Patients were classified according to the WHO criteria and staged according to the tumor-node-metastasis (TNM) classification (11). The present study was approved by the Ethics Committee of Dezhou People's Hospital (Dezhou, China) and written informed consent was obtained from all participants.

Cell culture and transfection. The human cervical cancer cell lines $\mathrm{SiHa}, \mathrm{HeLa}$, Caski, and C33a and the healthy cervical epithelial cell line End1/E6E7 were obtained from the Cell Bank of Chinese Academy of Sciences (Shanghai, China). All cell lines were cultured in Dulbecco's modified Eagle's medium (DMEM; Thermo Fisher Scientific, Inc., Waltham, MA, USA) with $10 \%$ fetal bovine serum (FBS; Thermo Fisher Scientific, Inc.) at $37^{\circ} \mathrm{C}$ in a humidified incubator containing $5 \% \mathrm{CO}_{2}$. Lipofectamine 2000 (Thermo Fisher Scientific, Inc.) was used to conduct cell transfection according to the manufacturer's instructions. The Caski cells were transfected with $100 \mathrm{nM}$ of miR-124-3p mimics to upregulate its expression, and cells transfected with $100 \mathrm{nM}$ of scramble miR (cat. no. yb1004; miR-NC) were used as control. The Caski cells were transfected with $100 \mathrm{nM}$ of miR-124-3p inhibitor to knock down its expression, and cells transfected with $100 \mathrm{nM}$ of negative control (NC) inhibitor were used as control. To study the function of miR-124-3p and IGF2BP1, Caski cells were co-transfected with $100 \mathrm{nM}$ of miR-124-3p mimic (cat. no. yb2033) and $5 \mu \mathrm{g}$ of blank pcDNA3.1 vector (cat. no. yb0086; miR-124-3p + blank) or $100 \mathrm{nM}$ of miR-124-3p mimic and $5 \mu \mathrm{g}$ of pcDNA3.1-IGF2BP1 plasmid (cat. no. yb0116; miR-124-3p + IGF2BP1), and cells co-transfected with $100 \mathrm{nM}$ miR-NC and $5 \mu \mathrm{g}$ of blank pcDNA3.1 vector (miR-NC + blank) were used as control. All these miR mimic, inhibitor and plasmid were purchased from Yearthbio (Changsha, China), and manufacturer did not supply the sequences. Analysis of gene expression was conducted $48 \mathrm{~h}$ following transfection.

Reverse transcription-quantitative polymerase chain reaction (RT-qPCR). TRIzol reagent (Thermo Fisher Scientific, Inc.) was used to extract total RNA from tissues and cell lines. RNA was then converted into cDNA using the RevertAid RT Reverse Transcription kit (Thermo Fisher Scientific, Inc.), which was performed in accordance with the manufacturer's instructions. A MirVana ${ }^{\mathrm{TM}}$ Real-Time RT-PCR MicroRNA Detection kit (Thermo Fisher Scientific, Inc.) was used to determine miR expression, following the manufacturer's instructions. U6 was used as an internal reference. mRNA expression was measured by qPCR using the Standard SYBR-Green RT-PCR kit (Takara Bio, Inc., Otsu, Japan) according to the manufacturer's instructions and GAPDH was used as an internal reference. The reaction conditions were $95^{\circ} \mathrm{C}$ for $3 \mathrm{~min}$, followed by 40 cycles of $95^{\circ} \mathrm{C}$ for $15 \mathrm{sec}$ and $60^{\circ} \mathrm{C}$ for $30 \mathrm{sec}$. The primer sequences were as follows: Insulin-like growth factor $2 \mathrm{mRNA}$ binding protein 1 (IGF2BP1) forward, 5'-CAAAGGAGCCGG AAAATTCAAAT-3' and reverse, 5'-CGTCTCACTCTCGGT GTTCA-3'; GAPDH forward, 5'-ACAACTTTGGTATCG TGGAAGG-3' and reverse, 5'-GCCATCACGCCACAGTTT C-3'; miR-124-3p forward, 5'-CGGGTAGCAGGCTTCTGA GT-3' and reverse, 5'-AAACCCCTCTCTGTCGGTAGCT-3'; U6 forward, 5'-CTCGCTTCGGCAGCACA-3' and reverse, 5'-AACGCTTCACGAAYYYGCGT-3'. Relative expression levels were quantified using the $2^{-\Delta \Delta C q}$ method (12).

Western blot analysis. Caski cells were lysed in Protein Lysis Buffer (Beyotime Institute of Biotechnology, Shanghai, China) following the manufacturer's instructions. Protein concentration was determined using the BCA Protein assay kit (Thermo Fisher Scientific, Inc.) following the manufacturer's instructions. For each well, protein $(60 \mu \mathrm{g})$ was separated using $10 \%$ SDS-PAGE and then transferred to a PVDF membrane (Thermo Fisher Scientific, Inc.). Following $3 \mathrm{~h}$ incubation at room temperature in TBST containing 5\% nonfat dried milk (Yili Group, Beijing, China), the PVDF membrane was incubated with primary antibodies against IGF2BP1 (cat. no. ab82968) and GAPDH (cat. no. ab9485) (1:100; both from Abcam, Cambridge, MA, USA) at room temperature for a further $3 \mathrm{~h}$ and then washed with TBST three times. Subsequently, the membrane was incubated with horseradish peroxidase-conjugated goat anti-rabbit secondary antibody (cat. no. ab97051, 1:5,000; Abcam) for $40 \mathrm{~min}$ at room temperature. The membrane was then washed three times with TBST and the immune complexes on the PVDF membrane were detected using the ECL Western Blotting kit (Thermo Fisher Scientific, Inc.), following the manufacturer's instructions. ImageJ software v.1.48 (National Institutes of Health, Bethesda, MD, USA) was used to analyze relative protein expression, which was presented as the density ratio versus GAPDH.

Cell proliferation analysis. Caski cells $\left(5 \times 10^{3}\right.$ cells/well $)$ were seeded in 96-well plates with $100 \mu \mathrm{l}$ fresh serum-free DMEM and $0.5 \mathrm{~g} / 1$ MTT (Sigma-Aldrich; Merck KGaA, Darmstadt, Germany). Following incubation at $37^{\circ} \mathrm{C}$ for 0 , 24, 48 and $72 \mathrm{~h}$, the medium containing MTT was removed and $50 \mu 1$ dimethyl sulfoxide (Sigma-Aldrich; Merck KGaA) was added. Following incubation at $37^{\circ} \mathrm{C}$ for $10 \mathrm{~min}$, the absorbance at $570 \mathrm{~nm}$ of each sample was determined using a plate reader.

Cell migration assay. Caski cells were cultured until 100\% confluence was reached, the wound was formed with a plastic scriber and cells were washed with Dulbecco's phosphate-buffered saline. Following this, Caski cells were cultured in DMEM with $10 \% \mathrm{FBS}$ at $37^{\circ} \mathrm{C}$ in a humidified atmosphere of $5 \% \mathrm{CO}_{2}$ for $48 \mathrm{~h}$ and then observed under a light microscope. 
Table I. Association between miR-124-3p expression and clinicopathological characteristics in cervical cancer.

\begin{tabular}{|c|c|c|c|c|}
\hline $\begin{array}{l}\text { Clinicopathological } \\
\text { characteristics }\end{array}$ & $\begin{array}{l}\text { Number } \\
(\mathrm{n}=85)\end{array}$ & $\begin{array}{l}\text { Low miR-124-3p } \\
\qquad(\mathrm{n}=41)\end{array}$ & $\begin{array}{l}\text { High miR-124-3p } \\
\qquad(\mathrm{n}=44)\end{array}$ & P-values \\
\hline Age (years) & & & & 0.658 \\
\hline$<55$ & 34 & 15 & 19 & \\
\hline$\geq 55$ & 51 & 26 & 25 & \\
\hline Tumor size $(\mathrm{cm})$ & & & & 0.114 \\
\hline$\leq 3$ & 57 & 31 & 26 & \\
\hline$>3$ & 28 & 10 & 18 & \\
\hline Differentiation & & & & $0.002^{\mathrm{a}}$ \\
\hline Well-moderate & 40 & 12 & 28 & \\
\hline Poor & 45 & 29 & 16 & \\
\hline Node metastasis & & & & $0.024^{\mathrm{a}}$ \\
\hline No & 32 & 10 & 22 & \\
\hline Yes & 53 & 31 & & \\
\hline Distant metastasis & & & & $0.003^{\mathrm{a}}$ \\
\hline No & 61 & 23 & 38 & \\
\hline Yes & 24 & 18 & 6 & \\
\hline Clinical stage & & & & $0.001^{\mathrm{a}}$ \\
\hline I-II & 31 & & 24 & \\
\hline III-IV & 54 & 34 & 20 & \\
\hline
\end{tabular}

Cell invasion assay. A Transwell assay was conducted to examine cell invasion using a 24-well Transwell chamber with a layer of Matrigel (Chemicon; EMD Millipore, Billerica, MA, USA). Caski cell suspension containing $5 \times 10^{5}$ cells was added to the upper chamber with DMEM and DMEM containing 10\% FBS was added to the lower chamber. Following $24 \mathrm{~h}$ incubation in a $37^{\circ} \mathrm{C}$ humidified atmosphere of $5 \% \mathrm{CO}_{2}$, cells on the interior of the inserts were removed using a cotton-tipped swab. Invading cells on the lower surface of the membrane were stained with gentian violet for $10 \mathrm{~min}$ at room temperature (Sigma-Aldrich; Merck KGaA), rinsed with water, dried in air and counted under a light microscope.

Bioinformatical predication and luciferase reporter gene assay. Targetscan (www.targetscan.org/) was used to predicate the putative target genes of miR-124-3p. The wild-type (WT) of IGF2BP1 3'UTR was constructed by YearthBio (Changsha, China) and inserted into the pMIR-REPORT miRNA Expression Reporter vector (Thermo Fisher Scientific, Inc.) generating the WT-IGF2BP1-3'UTR plasmid. The mutant type (MT) of IGF2BP1 3'UTR was constructed using the Easy Mutagenesis System kit (Promega Corporation, Madison, WI, USA), in accordance with the manufacturer's instructions. This was also inserted into the pMIR-REPORT miRNA Expression Reporter vector to generate the MT-IGF2BP1-3'UTR plasmid. Caski cells were co-transfected with WT-IGF2BP1-3'UTR plasmid, or MT-IGF2BP1-3'UTR plasmid and miR-NC or miR-423-3p mimic using Lipofectamine 2000. Following transfection for $48 \mathrm{~h}$, luciferase activity was determined using the dual-luciferase reporter assay system (Promega Corporation) and Renilla luciferase activity was normalized to firefly luciferase activity.

Statistical analysis. The data were expressed as the mean \pm standard deviation. Statistical analysis was performed using SPSS 20.0 (IBM Corp, Armonk, NY, USA). The differences between the two groups were analyzed using a Student's t-test. Pearson's correlation coefficient was also used. The association between miR-124-3p expression and the clinical characteristics of cervical cancer was analyzed using a $\chi^{2}$ test. $\mathrm{P}<0.05$ was considered to indicate a statistically significant difference.

\section{Results}

Downregulation of miR-124-3p is associated with cervical cancer progression. miR-124-3p expression was examined in cervical cancer tissues and matching adjacent non-tumor tissues. The results of RT-qPCR indicated that levels of miR-124-3p levels were significantly reduced in cervical cancer tissues compared with matching adjacent non-tumor tissues $(\mathrm{P}<0.0001$; Fig. 1A). Subsequently, miR-124-3p levels in the cervical cancer cell lines SiHa, HeLa, Caski and C33a as well as in normal cervical epithelial cell line End1/E6E7 were examined. It was demonstrated that miR-124-3p levels were significantly downregulated in cervical cancer cell lines compared with End1/E6E7 cells (all $\mathrm{P}<0.01$; Fig. 1B).

The clinical significance of miR-124-3p in cervical cancer was then investigated. Patients with cervical cancer were 


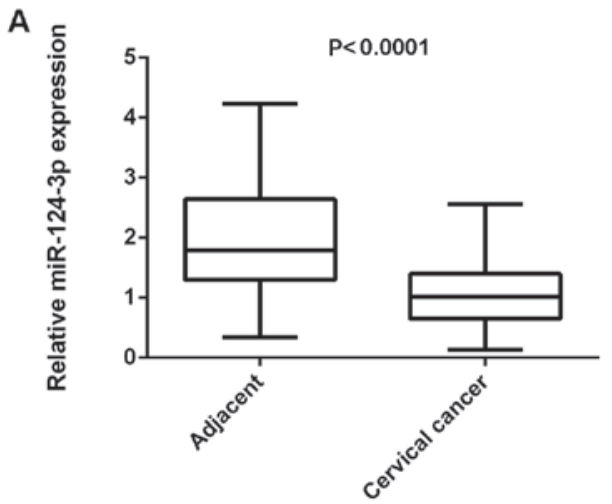

B

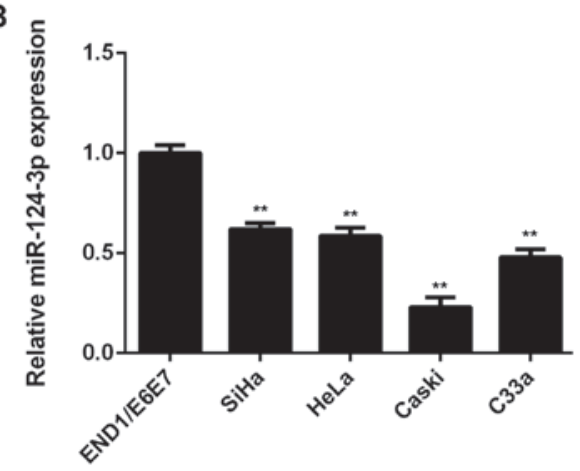

Figure 1. Downregulation of miR-124-3p is associated with cervical cancer progression. (A) RT-qPCR was used to determine miR-124-3p expression in cervical cancer tissues compared with matching adjacent non-tumor tissues. (B) RT-qPCR was used to determine miR-124-3p expression in cervical cancer cell lines compared with the normal human cervical epithelial cell line END1/E6E7. * P<0.01 vs. END1/E6E7. miR, microRNA; RT-qPCR, reverse transcription-quantitative polymerase chain reaction.

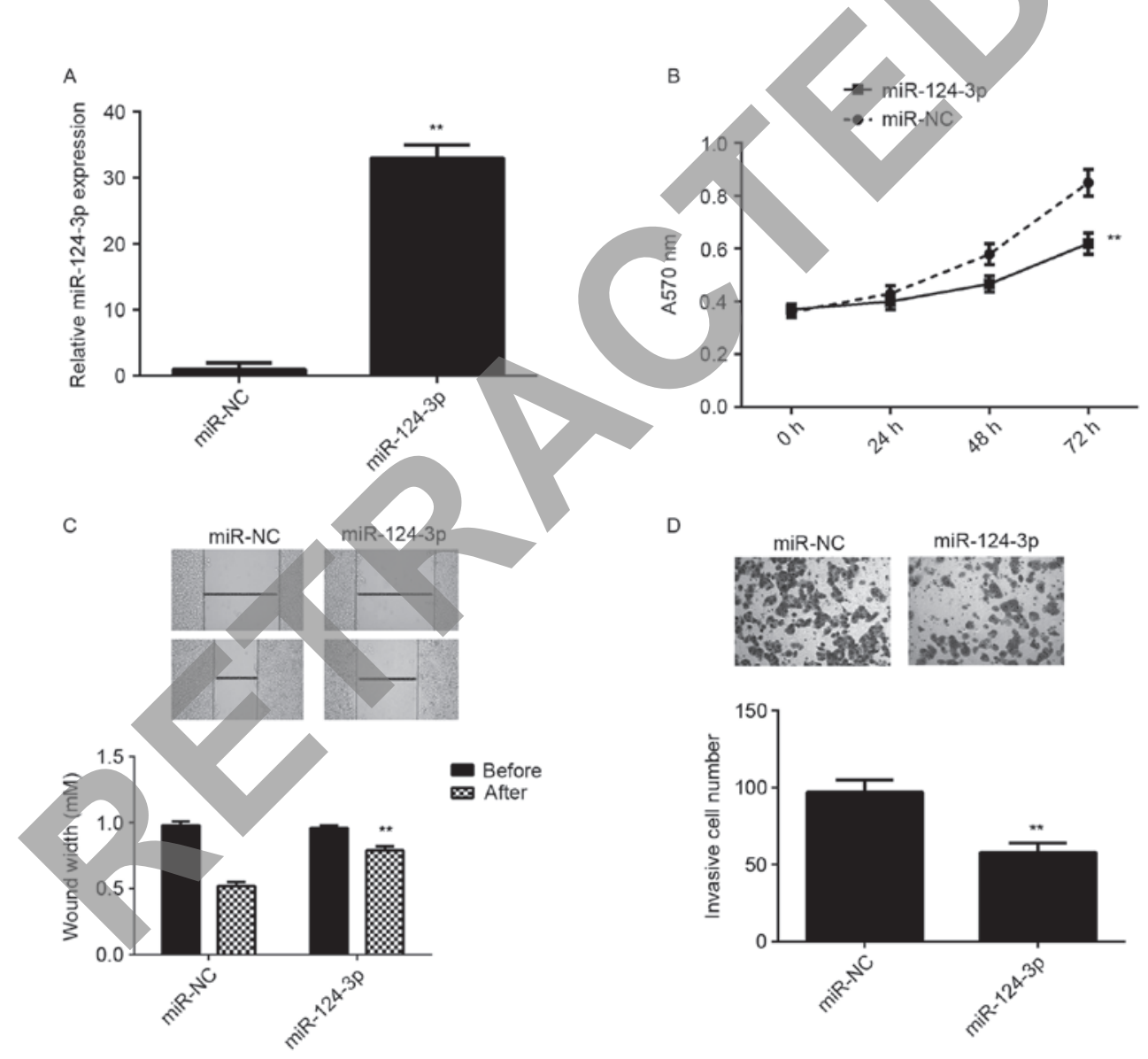

Figure 2. miR-124-3p overexpression inhibits Caski cell proliferation, migration and invasion. Caski cells were transfected with miR-124-3p mimic or miR-NC. (A) Reverse transcription-quantitative polymerase chain reaction was used to measure miR-124-3p expression. (B) MTT, (C) wound healing and (D) Transwell assays were used to determine cell proliferation, migration and invasion, respectively. ${ }^{* *} \mathrm{P}<0.01 \mathrm{vs}$. miR-NC. The magnification for the wound healing assay was $\mathrm{x} 40$ and for the Transwell assay it was $\mathrm{x} 400$. miR, microRNA; NC, negative control; A570, absorbance at $570 \mathrm{~nm}$.

divided into two groups based on the mean value of miR-124-3p expression. Low expression of miR-124-3p was significantly associated with poor differentiation, metastasis and advanced clinical stage in cervical cancer (all $\mathrm{P}<0.01$; Table I). These results suggest that the downregulation of miR-124-3p may contribute to the progression of cervical cancer.

Ectopic expression of miR-124-3p inhibits the proliferation, migration and invasion of Caski cells. Caski cells exhibited the greatest reduction in miR-124-3p expression compared with the End1/E6E7 cells (Fig. 1A), therefore this cell line was used in subsequent experiments. To determine the role of miR-124-3p in cervical cancer, Caski cells were transfected with miR-124-3p or miR-NC. The results of RT-qPCR indicated that miR-124-3p levels were significantly higher in the miR-124-3p group compared with the miR-NC group $(\mathrm{P}<0.01$; Fig. 2A). MTT, wound healing and Transwell assays were all performed to determine cell proliferation, migration and 
A
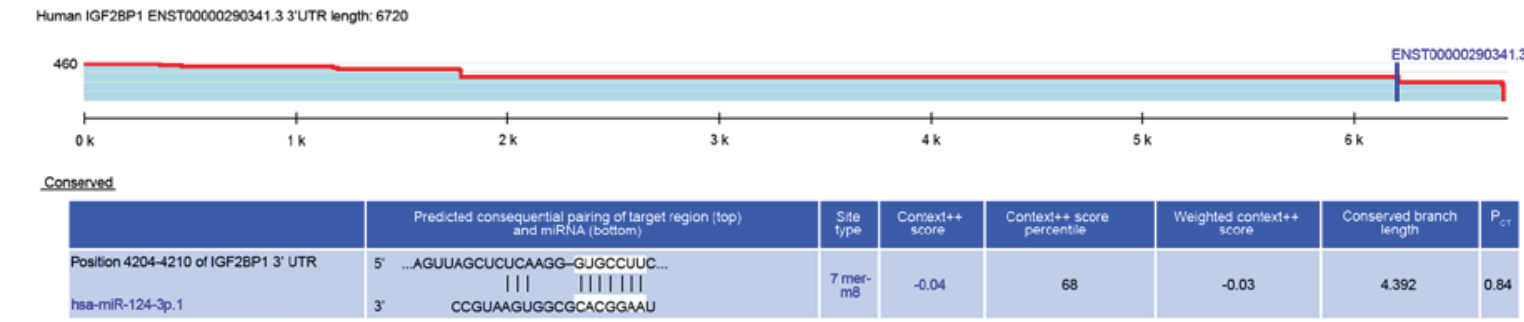

B
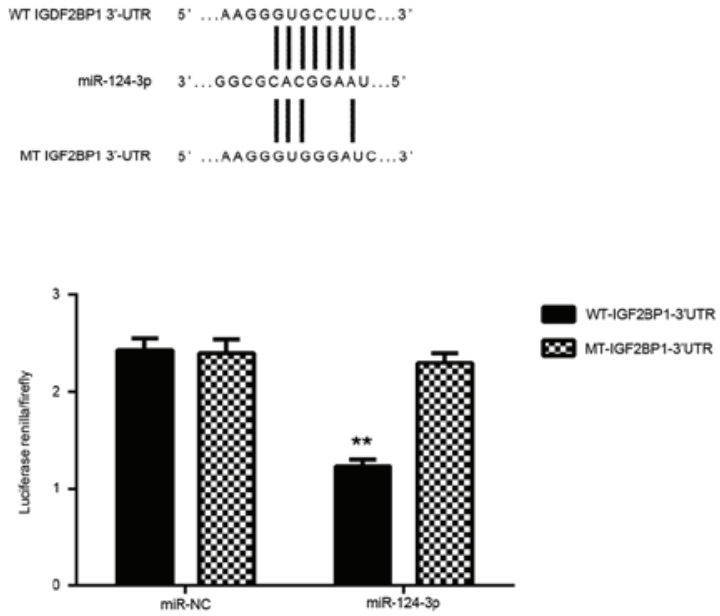

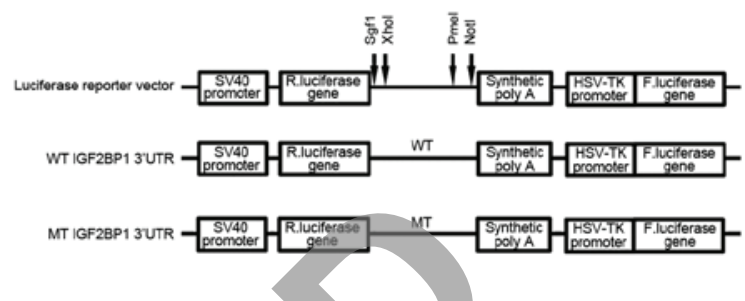

Figure 3. IGF2BP1 is a target of miR-124-3p in Caski cells. (A) Targetscan indicated that IGF2BP1 was a putative target of miR-124-3p. (B and C) Luciferase reporter vectors containing WT or MT IGF2BP1 3'UTR were constructed. (D) A luciferase reporter assay was conducted in Caski cells. ${ }^{* *} \mathrm{P}<0.01$ vs. miR-NC. miR, microRNA; WT, wild-type; MT, mutant type; UTR, untranslational region; NC, negative control.

invasion, respectively. It was demonstrated that the overexpression of miR-124-3p significantly reduced the proliferation, migration and invasion of Caski cells compared with the miR-NC group (all $\mathrm{P}<0.01$; Fig. 2B-D). These results suggest that miR-124-3p suppresses the growth and metastasis of cervical cancer.

IGF2BP1 is a target gene of miR-124-3p in Caski cells. The putative target genes of miR-124-3p were analyzed. Targetscan data indicated that IGF2BP1 was a potential target gene of miR-124-3p (Fig. 3A). To confirm this targeting association, luciferase vectors containing WT or MT IGF2BP1 3'UTR were constructed (Fig. 3B and C). A luciferase reporter gene assay was then conducted and it was determined that luciferase activity was significantly reduced in Caski cells co-transfected with miR-124-3p mimics and WT-IGF2BP1-3'UTR plasmid compared with the control group $(\mathrm{P}<0.01$; Fig. 3D). This reduction was eliminated when cells were transfected with a MT-IGF2BP1-3'UTR plasmid (Fig. 3D). This indicates that IGF2BP1 may be a novel target gene of miR-124-3p.

Upregulation of IGF2BPI is inversely correlated with miR-124-3p expression in cervical cancer. To determine the role of IGF2BP1 in cervical cancer, the expression of IGF2BP1 in cervical cancer tissues was measured. Levels of IGF2BP1 mRNA were significantly increased in cervical cancer tissues compared with matched adjacent non-tumor tissues $(\mathrm{P}<0.0001$; Fig. 4A). Furthermore, levels of IGF2BP1 mRNA and protein were higher in the cervical cancer cell lines compared with End1/E6E7 cells (all $\mathrm{P}<0.01$; Fig. 4B and $\mathrm{C}$ ). In addition, IGF2BP1 levels were inversely correlated with miR-124-3p levels in cervical cancer tissues ( $r=-0.744$; Fig. 4D). These results suggest that the upregulation of IGF2BP1 may be caused by the downregulation of miR-124-3p in cervical cancer tissues.

miR-124-3p negatively regulates the protein expression of IGF2BP1 in Caski cells. The effects of miR-124-3p on the expression of IGF2BP1 protein in Caski cells were investigated. It was demonstrated that the expression of IGF2BP1 protein was significantly lower in the miR-124-3p group compared with the miR-NC group ( $\mathrm{P}<0.01$; Fig. 5A), indicating that overexpression of miR-124-3p inhibits the expression of IGF2BP1. To further confirm these findings, Caski cells were transfected with either miR-124-3p inhibitor or NC inhibitor. Following transfection, levels of miR-124-3p were significantly reduced in the miR-124-3p inhibitor group compared with the NC inhibitor group $(\mathrm{P}<0.01$; Fig. $5 \mathrm{~B})$. Furthermore, levels of IGF2BP1 protein were higher in the miR-124-3p inhibitor group compared with the $\mathrm{NC}$ inhibitor group, indicating that inhibition of miR-124-3p significantly promotes the protein expression of IGF2BP1 ( $<<0.01$; Fig. 5C). These results demonstrate that miR-124-3p negatively regulates the expression of IGF2BP1 in Caski cells.

Overexpression of IGF $2 B P 1$ attenuates the effects of miR-124-3p on the malignant phenotypes of Caski cells. 


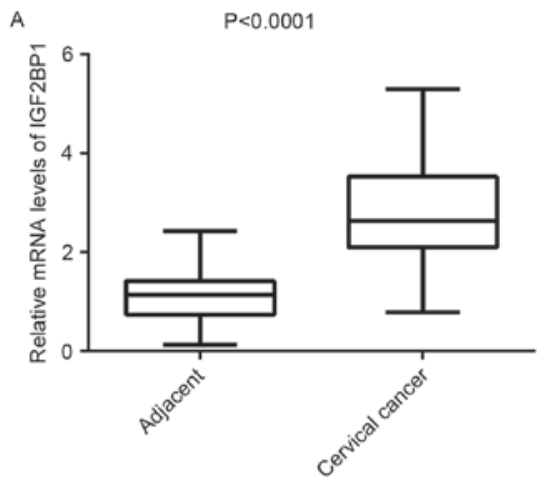

B
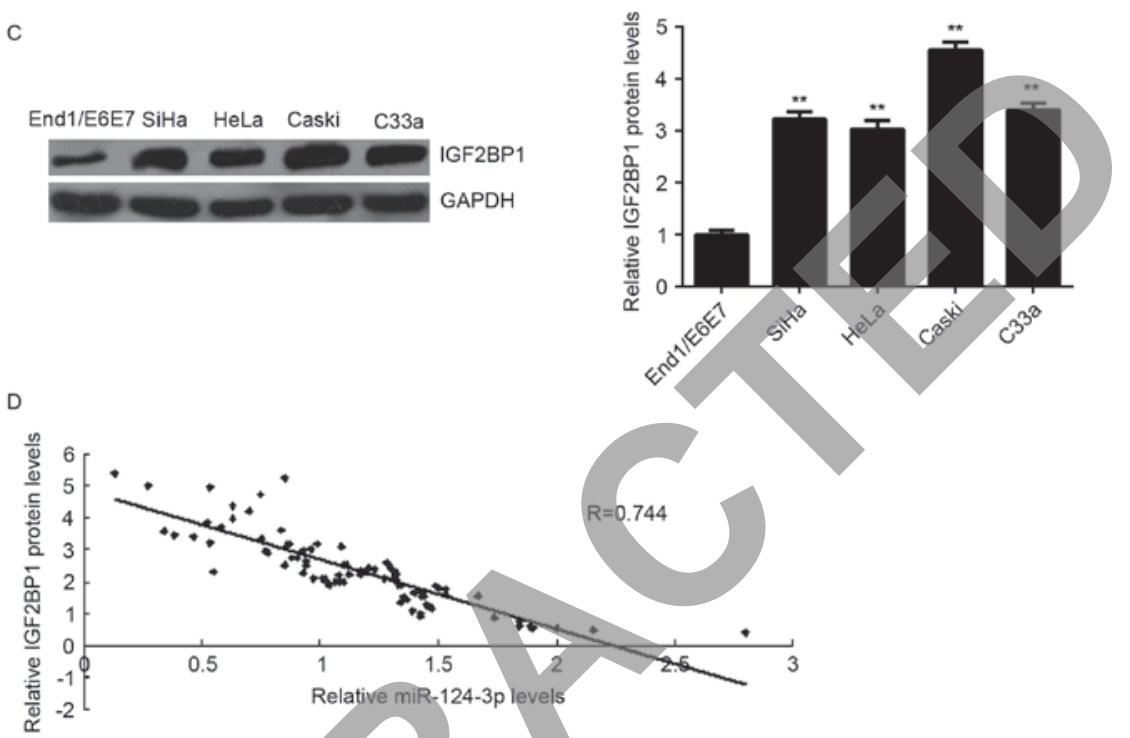

Figure 4. Upregulation of IGF2BP1 inversely correlates with miR-124-3p levels in cervical cancer. RT-qPCR was used to determine levels of IGF2BP1 mRNA in cervical cancer tissues compared with matched adjacent non-tumor tissues. (B) RT-qPCR and (C) western blot analysis were used to measure mRNA and protein levels of IGF2BP1 in the cervical cancer cell lines compared with normal human cervical epithelial END1/E6E7 cells. *P $<0.01$ vs. END1/E6E7. (D) An inverse correlation was identified between levels of IGF2BP1 mRNA and miR-124-3p in cervical cancer tissues. miR, microRNA; RT-qPCR, reverse transcription-quantitative polymerase chain reaction; IGFBP1, insulin-like growth factor 2 mRNA binding protein 1.

IGF2BP1 was upregulated in cervical cancer and its expression was negatively regulated by miR-124-3p in Caski cells, therefore, it was hypothesized that IGF2BP1 may be involved in the development of the miR-124-3p-mediated malignant phenotypes of Caski cells. Thus, Caski cells were co-transfected with scramble miR and blank pcDNA3.1 vector, miR-124-3p mimic and a blank pcDNA3.1 vector and miR-124-3p mimic pcDNA3.1-IGF2BP1 plasmid, respectively. Following transfection, it was determined that levels of IGF2BP1 were significantly reduced in the miR-124-3p + blank group compared with the miR-NC + blank group; however, the levels of IGF2BP1 protein were higher in the miR-124-3p + IGF2BP1 group compared with the miR-124-3p + blank group (Fig. 6A). MTT, wound healing and Transwell assays were subsequently performed. The proliferation, migration and invasion of Caski cells were significantly decreased in the miR-124-3p + blank group compared with miR-NC + blank group. However proliferation, migration and invasion of Caski cells were significantly increased in the miR-124-3p + IGF2BP1 group compared with the miR-124-3p + blank group ( $\mathrm{P}<0.01$; Fig. 6B-D). These results suggest that overexpression of IGF2BP1 attenuates the suppressive effects of miR-124-3p on the malignant phenotypes of Caski cells.

\section{Discussion}

It has been demonstrated that miR-124-3p serves a suppressive role in cervical cancer (7). However, the detailed molecular mechanism by which miR-124-3p affects cervical cancer growth and metastasis remains largely unclear. The results of the present study demonstrated that miR-124-3p was significantly downregulated in cervical cancer and that the decreased expression of miR-124-3p was associated with its malignant progression, as well as the poor prognosis of patients with cervical cancer. The ectopic expression of miR-124-3p suppressed the proliferation, migration and invasion of Caski cells. IGF2BP1 was identified as a novel target of miR-124-3p in Caski cells and overexpression of IGF2BP1 attenuated the suppressive effects of miR-124-3p on the malignant phenotypes of Caski cells. IGF2BP1 was upregulated in cervical cancer and was inversely correlated with miR-124-3p levels in cervical cancer tissues.

It was demonstrated that miR-124-3p acts as a tumor suppressor by regulating different targets in common cancers. For example, the downregulation of miR-124-3p is implicated in the tumorigenesis of gastric cancer (13). $\mathrm{Xu}$ et al (14) reported that miR-124-3p inhibits the migration 

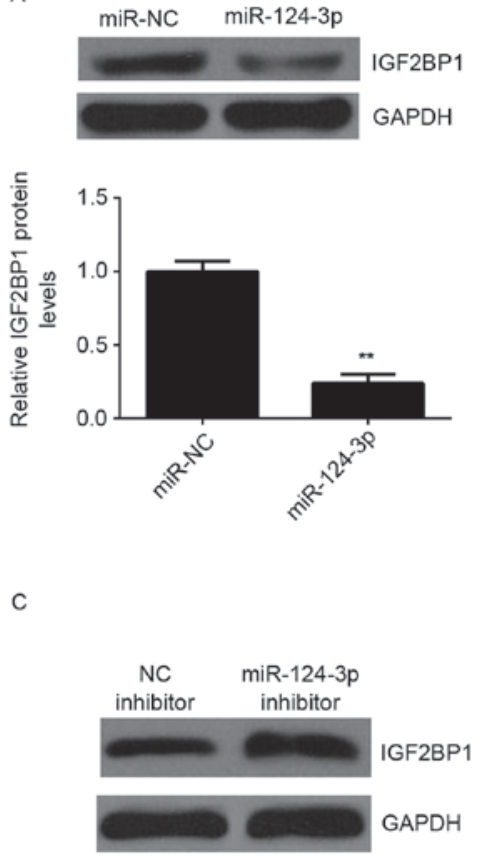

B
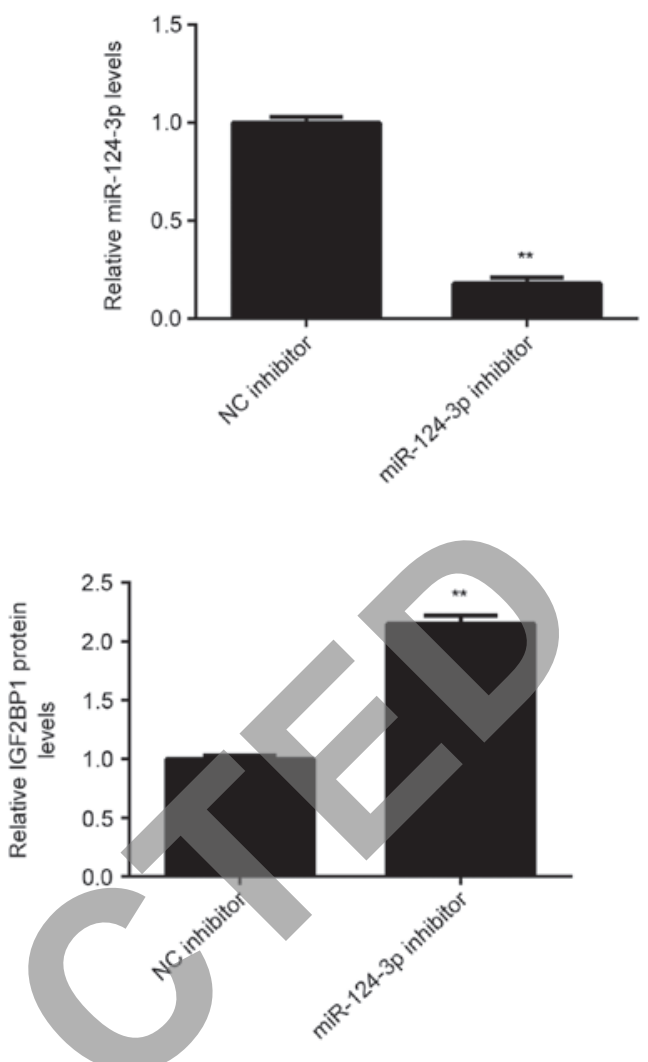

Figure 5. Protein expression of IGF2BP1 is negatively regulated by miR-124-3p in Caski cells. (A) Western blot analysis was used to detect the protein expression of IGF2BP1 in Caski cells transfected with miR-124-3p mimic or miR-NC, respectively. ${ }^{* *} \mathrm{P}<0.01$ vs. miR-NC. Caski cells were transfected with miR-124-3p inhibitor or NC inhibitor, respectively. (B) Reverse transcription-quantitative polymerase chain reaction was used to measure miR-124-3p levels and $(\mathrm{C})$ western blot analysis was conducted to measure the protein expression of IGF2BP1. ${ }^{* *} \mathrm{P}<0.01 \mathrm{vs.} \mathrm{NC}$ inhibitor. miR, microRNA; NC, negative control; IGF2BP1, insulin-like growth factor-2 mRNA binding protein-1,

and invasion of bladder cancer cells by targeting ROCK1. Similarly, miR-124-3p suppressed cell proliferation, invasion and aerobic glycolysis and promoted cell apoptosis in astrocytoma (15). Furthermore, miR-98 may serve a suppressive role in cervical cancer. Wilting et al (7) investigated the methylation status of miR-124 in 139 cervical tissue specimens and observed that methylation frequency increased from $0 \%$ in normal tissues to $93 \%$ in cervical carcinomas, resulting in a reduction in the expression of miR-124. In the present study, miR-124-3p was significantly downregulated in cervical cancer tissues and cell lines compared with matching adjacent non-tumor tissues and normal cervical epithelial cells, respectively. Furthermore, it was observed that low expression of miR-124-3p was associated with metastasis and a more advanced clinical stage of cervical cancer, suggesting that the downregulation of miR-124-3p may contribute to the progression of cervical cancer. The present study also indicated that overexpression of miR-124-3p significantly reduced the proliferation, migration and invasion of Caski cells. Previous studies have reported that miR-124 suppresses the proliferation, migration and invasion of cervical cancer cells $(8,10)$, which is consistent with the results of the present study. The rs531564 polymorphism in miR-124 may also affect the genetic susceptibility of individuals to cervical cancer. A comparison between those carrying the $\mathrm{CC}$ genotype and individuals carrying $\mathrm{CG}$ and GG genotypes demonstrated that those harboring the CC gene exhibited a significantly reduced risk of developing cervical cancer (16).

miRs function by negatively regulating the expression of their targets (17) therefore a bioinformatics analysis was performed in the present study to predicate the potential target genes of miR-124-3p. Targetscan data indicated that IGF2BP1 was a putative target gene of miR-124-3p. Luciferase reporter gene assay data confirmed that miR-124-3p could directly bind to the 3'UTR of IGF2BP1 mRNA and it was concluded that IGF2BP1 is a novel target gene of miR-124-3p. Furthermore, the expression of IGF2BP1 was negatively regulated by miR-124-3p in Caski cells. IGF2BP1 belongs to the IGF2BP family and contains four K homology domains and two RNA recognition motifs (17). IGF2BP1 can directly bind to the mRNAs of certain genes including insulin-like growth factor $2, \beta$-actin and $\beta$-transducing repeat-containing protein and therefore induces their translation $(18,19)$. It was recently reported that IGF2BP1 serves an oncogenic role in cervical cancer (20). Su et al (20) demonstrated that miR-140-5p suppressed cervical cancer growth and metastasis by targeting IGF2BP1. However, the detailed regulatory mechanism underlying IGF2BP1 expression in cervical cancer remains unclear. In the present study, it was determined that the overexpression of IGF2BP1 attenuates the suppressive effects of miR-124-3p upregulation on the 
A
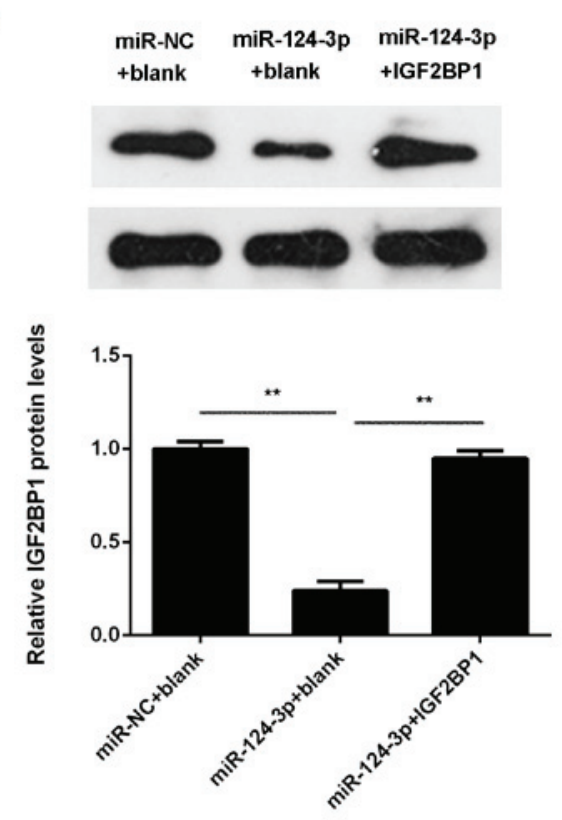

B
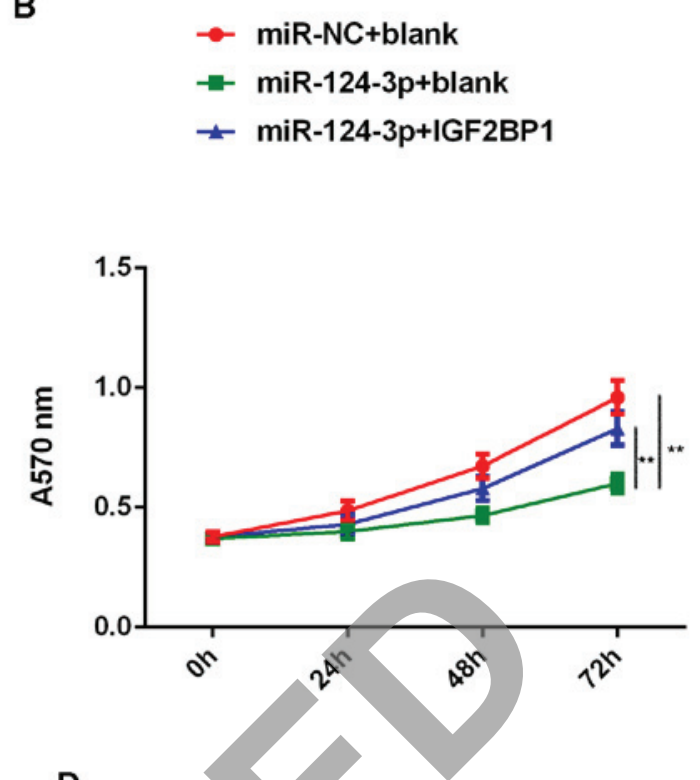

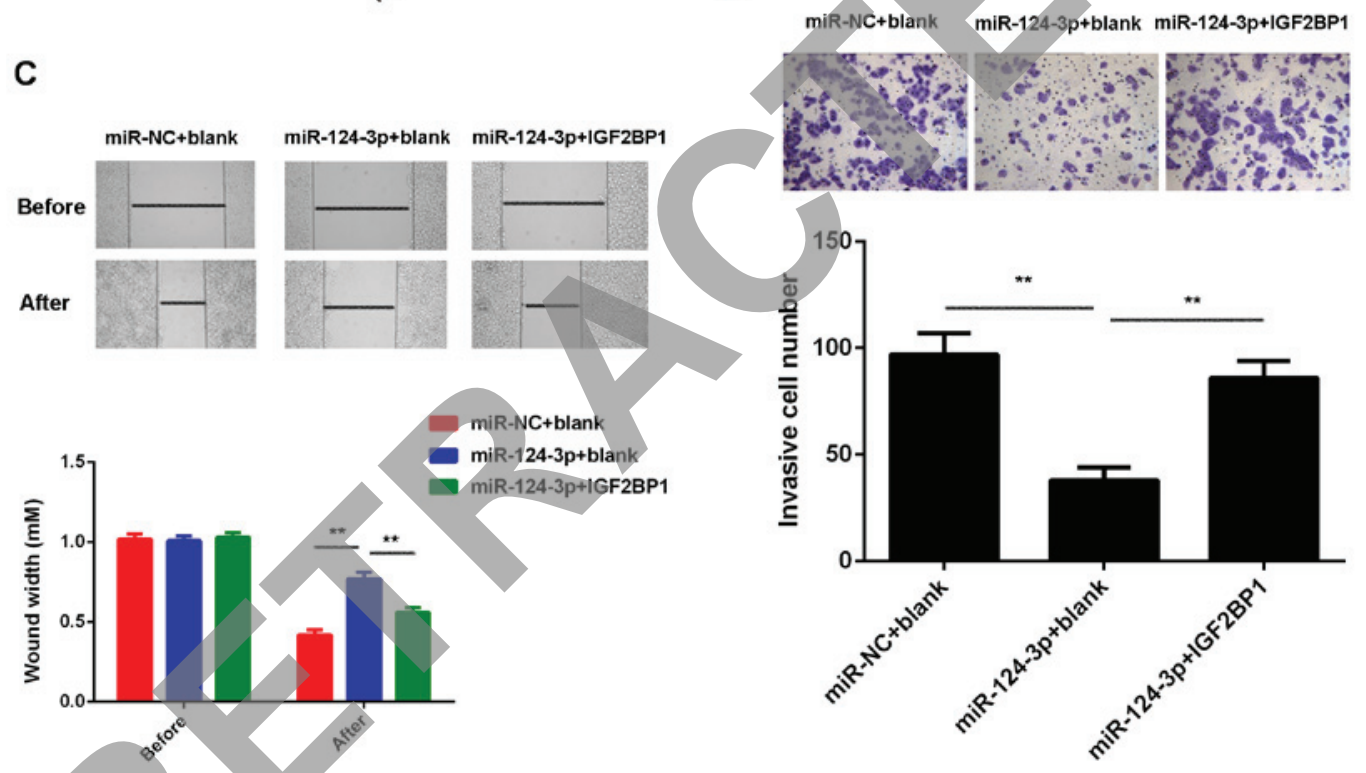

Figure 6. Overexpression of IGF2BP1 attenuates the suppressive effects of miR-124-3p on the malignant phenotypes of cervical cancer cells. Caski cells were co-transfected with miR-NC and blank pcDNA3.1 vector (miR-NC + blank), miR-124-3p mimic and blank pcDNA3.1 vector (miR-124-3p + blank) and miR-124-3p mimic pcDNA3.1-IGF2BP1 plasmid (miR-124-3p + IGF2BP1), respectively. (A) Western blot analysis was conducted to examine the expression of IGF2BP1 protein. (B) MTT, (C) wound healing and (D) Transwell assays were conducted to determine cell proliferation, migration and invasion, respectively. ${ }^{* *} \mathrm{P}<0.01$. The magnification for the wound healing assay was $\mathrm{x} 40$ and for the Transwell assay it was $\mathrm{x} 400$. miR, microRNA; NC, negative control; A570, absorbance at $570 \mathrm{~nm}$; IGF2BP1, insulin-like growth factor-2 mRNA binding protein-1.

proliferation, migration and invasion of Caski cells. This suggests that miR-124-3p inhibits cell proliferation and induces cell apoptosis in cervical cancer by directly targeting IGF2BP1. In addition the present study demonstrated that IGF2BP1 was significantly upregulated in cervical cancer tissues and cell lines and that levels of IGF2BP1 mRNA were inversely correlated with levels of miR-124-3p in cervical cancer tissues. These results suggest that the upregulation of IGF2BP1 may be due to the downregulation of miR-124-3p in cervical cancer.

Various other targets of miR-124 have been identified in cervical cancer. For example, Wan et al (10) reported that miR-124 repressed vasculogenic mimicry and cell motility in cervical cancer cells by targeting AmotL1. Additionally,
Zhang et al (8) demonstrated that miR-124 inhibited the proliferation, invasion, migration and EMT of cervical carcinoma cells by targeting the astrocyte-elevated gene-1. Liu et al (9) indicated that MALAT1 may promote the proliferation and invasion of HR-HPV(+) cervical cancer cells, at least partially, by inhibiting the expression of miR-124, thus upregulating the expression of its target gene RBG2.

In conclusion, the present study is the first to demonstrate that miR-124-3p is downregulated in cervical cancer and inhibits the proliferation, migration and invasion of cervical cancer cells by inhibiting the expression of its novel identified target gene IGF2BP1. This suggests that whilst IGF2BP1 remains a therapeutic target, miR-124-3p may be a potential candidate for the treatment of cervical cancer. 


\section{References}

1. Torre LA, Bray F, Siegel RL, Ferlay J, Lortet-Tieulent J and Jemal A: Global cancer statistics, 2012. CA Cancer J Clin 65: 87-108, 2015

2. Wright JD, Huang Y, Ananth CV, Tergas AI, Duffy C, Deutsch I, Burke WM, Hou JY, Neugut AI and Hershman DL: Influence of treatment center and hospital volume on survival for locally advanced cervical cancer. Gynecol Oncol 139: 506-512, 2015.

3. Liu L, Yu X, Guo X, Tian Z, Su M, Long Y, Huang C, Zhou F, Liu M, Wu X and Wang X: miR-143 is downregulated in cervical cancer and promotes apoptosis and inhibits tumor formation by targeting Bcl-2. Mol Med Rep 5: 753-760, 2012.

4. Yao J, Deng B, Zheng L, Dou L, Guo Y and Guo K: miR-27b is upregulated in cervical carcinogenesis and promotes cell growth and invasion by regulating CDH11 and epithelial-mesenchymal transition. Oncol Rep 35: 1645-1651, 2016.

5. An L, Liu Y, Wu A and Guan Y: microRNA-124 inhibits migration and invasion by down-regulating ROCK1 in glioma. PLoS One 8: e69478, 2013.

6. Han G, Wang Y, Bi W, Jia J and Wang W: MicroRNA-124 functions as a tumor suppressor and indicates prognosis in human osteosarcoma. Exp Ther Med 9: 679-684, 2015.

7. Wilting SM, van Boerdonk RA, Henken FE, Meijer CJ, Diosdado B, Meijer GA, le Sage C, Agami R, Snijders PJ and Steenbergen RD: Methylation-mediated silencing and tumour suppressive function of hsa-miR-124 in cervical cancer. Mol Cancer 9: 167, 2010.

8. Zhang X, Cai D, Meng L and Wang B: MicroRNA-124 inhibits proliferation, invasion, migration and epithelial-mesenchymal transition of cervical carcinoma cells by targeting astrocyte-elevated gene-1. Oncol Rep 36: 2321-2328, 2016.

9. Liu S, Song L, Zeng S and Zhang L: MALAT1-miR-124-RBG2 axis is involved in growth and invasion of HR-HPV-positive cervical cancer cells. Tumour Biol 37: 633-640, 2016.

10. Wan HY, Li QQ, Zhang Y, Tian W, Li YN, Liu M, Li X and Tang H: miR-124 represses vasculogenic mimicry and cell motility by targeting amotL1 in cervical cancer cells. Cancer Lett 355: 148-158, 2014.
11. Meva J, Chaudhary RK, Bhaduri D, Bhatia M, Hatti S and Ba R: Lacunae in international federation of gynecology and obstetrics (FIGO) classification for cervical carcinoma: Observational study using TNM classification as comparator. Int J Gynecol Cancer 23: 1071-1077, 2013.

12. Livak KJ and Schmittgen TD: Analysis of relative gene expression data using real-time quantitative PCR and the 2(-Delta Delta C(T)) method. Methods 25: 402-408, 2001.

13. Li H, Xie S, Liu M, Chen Z, Liu X, Wang L, Li D and Zhou Y: The clinical significance of downregulation of mir-124-3p, mir-146a-5p, mir-155-5p and mir-335-5p in gastric cancer tumorigenesis. Int J Oncol 45: 197-208, 2014.

14. Xu X, Li S, Lin Y, Chen H, Hu Z, Mao Y, Xu X, Wu J, Zhu Y, Zheng $\mathrm{X}$, et al: MicroRNA-124-3p inhibits cell migration and invasion in bladder cancer cells by targeting ROCK1. J Transl Med 11: 276, 2013.

15. Deng D, Wang L, Chen Y, Li B, Xue L, Shao N, Wang Q, Xia X, Yang Y and Zhi F: MicroRNA-124-3p regulates cell proliferation, invasion, apoptosis, and bioenergetics by targeting PIM1 in astrocytoma. Cancer Sci 107: 899-907, 2016.

16. Wu $\mathrm{H}$ and Zhang J: miR-124 rs531564 polymorphism influences genetic susceptibility to cervical cancer. Int J Clin Exp Med 7: 5847-5851, 2014

17. Ambros V: The functions of animal microRNAs. Nature 431: 350-355, 2004.

18. Bell JL, Wächter K, Mühleck B, Pazaitis N, Köhn M, Lederer M and Hüttelmaier $S$ : Insulin-like growth factor 2 mRNA-binding proteins (IGF2BPs): Post-transcriptional drivers of cancer progression? Cell Mol Life Sci 70: 2657-2675, 2013.

19. Luo Y, Sun R, Zhang J, Sun T, Liu X and Yang B: miR-506 inhibits the proliferation and invasion by targeting IGF2BP1 in glioblastoma. Am J Transl Res 7: 2007-2014, 2015.

20. Su Y, Xiong J,Hu J, Wei X,Zhang X and RaoL: MicroRNA-140-5p targets insulin like growth factor 2 mRNA binding protein 1 (IGF2BP1) to suppress cervical cancer growth and metastasis Oncotarget 7: 68397-68411, 2016.

This work is licensed under a Creative Commons Attribution-NonCommercial-NoDerivatives 4.0 International (CC BY-NC-ND 4.0) License. 